\title{
A conservative and entropy scheme for a simplified model of granular media
}

\author{
C. Buet $^{1}$, S. Cordier ${ }^{2}$, V. Dos Santos ${ }^{3}$
}

1: CEA,91680 Bruyères le Chatel, France.

email: christophe.buet@cea.fr

2: Laboratoire MAPMO,UMR 6628

Université Orléans, 45067 Orleans, France

email: stephane.cordier@univ-orleans.fr

3: Laboratoire Vision et Robotique,

IUT de Bourges,

18 Bourges, France

email: Valerie.Dossantos@bourges.univ-orleans.fr

\begin{abstract}
In this paper, we present a numerical scheme for a non linear FokkerPlanck equation of one-dimensional granular medium. We consider a kinetic description of a system of particles undergoing nearly elastic particles and interacting with a thermal bath. We construct a numerical method which preserve all the properties of the continuous model, conservation laws and decay of the entropy. Moreover the discretization is such that, on a fixed grid, we deal with arbitrary small temperatures for the bath. Explicit and implicit time discretization are analyzed
\end{abstract}




\section{Introduction}

The model we consider has been proposed by Mac Namara and Young [23], and has been studied from mathematical point of view ([5], [4], [3]). We also refer to $([1,17])$ for kinetic modelisations of inelastic collisions. The model is derived from a system of particles moving in one dimension and that undergo inelastic collision. The unknown of the kinetic model is the distribution function that represents the number of particles with velocity $v \in \mathbb{R}$, at time $t$. The collisions are modeled by a Boltzmann type collision operator, for inelastic collision with an hard sphere cross section. The collisions being inelastic, they lead to a decay of energy and the distribution function concentrates for large time on zero velocity. When there is a lot $(N \gg 1)$ of such collision, weakly inelastic, the Boltzmann equation reduces to a Fokker-Planck type equation, $[24,25]$.

In this work, we consider such a system of particles immersed in a thermal bath at constant temperature $\sigma$, which counterbalances the loss of energy due to inelastic collisions. When $\sigma=0$, the distribution function converges to a Dirac mass at origin in the sense of weak convergence of measure [3]. In the case $\sigma>0$, the equilibrium states behave like $\exp \left(-|v|^{3}\right)$ for large velocities, [4]: the equilibrium state are not Maxwellian.

Let us also mention that some works has been devoted to the hydrodynamical limit of this system: one obtains a system of 2 conservation laws for the density $\rho$ and the momentum $\rho u$, that can be written as the Euler system for isotropic gases, with a pressure law $P(\rho)=\rho^{\gamma}, \gamma=1 / 3$ (the exponent $1 / 3$ yields to mathematical difficulties since the obtained system lies in between classical gas dynamics, $\gamma>1$, and pressure less gas, $\gamma=0$, that have very different behavior).

The aim of this paper is to present a deterministic discretization that is compatible with the known properties of the operator. Moreover, the method is such that we can treat any temperature of the bath with the same grid, in other words, as $\sigma=0$ goes to zero the scheme degenerate to a conservative and entropy discretization of the pure granular case. The method is close to the one developed for the Fokker-Planck-Landau equation or the Kompaneets equation (Cf. Buet and al, [7, 9, 10]). For generalized discrete models of kinetic equations, we refer to ([20]). Let us emphasize that the proposed method has a linear computational cost. Explicit and Implicit time discretization are detailed. Implicit schemes appear to be more efficient.

Some numerical results are presented. In particular, the non-Maxwellian equilibrium states are obtained.

\section{The one dimensional model of granular me- dia}

We consider a simplified 1D model of granular media described by example in [4]. The particles are described by their distribution function $f(v, t)$ of velocity 
$v$ and time $t$. This function obeys

$$
\partial_{t} f=\partial_{v}\left(F f+\sigma \partial_{v} f\right)
$$

where $F f$ is the pure granular term,

$$
F(v)=\int_{\mathbb{R}}\left|v-v^{\prime}\right|\left(v-v^{\prime}\right) f\left(v^{\prime}\right) d v^{\prime},
$$

and, $\sigma$ is an arbitrary positive constant related to the temperature of the bath. All the more, $\sigma \partial_{v}^{2} f$ represents the thermal reservoir, where $\sigma$ is linked to the temperature. Let us define $\rho$ and $u_{f}$ as the mass and the mean velocity respectively

$$
\rho=\int_{\mathbb{R}} f\left(v^{\prime}\right) d v^{\prime}, u_{f}=1 / \rho \int_{\mathbb{R}} f\left(v^{\prime}\right) v^{\prime} d v^{\prime} .
$$

\subsection{Properties of the continuous model}

The properties of this model are the conservation of mass and momentum, the decay of the energy for $(\sigma=0)$ and of the entropy:

Definition 2.1 Let us define the temperature and the entropy by respectively

$$
\begin{gathered}
T(f)=\int_{v^{\prime}}\left|v-u_{f}\right|^{2} f(v) d v \\
\mathcal{E}=\int \sigma[\ln (f(v))] f(v) d v+\frac{1}{6} \int_{v} \int_{v^{\prime}}\left|v-v^{\prime}\right|^{3} f\left(v^{\prime}\right) f(v) d v^{\prime} d v,
\end{gathered}
$$

and let be $E_{\alpha}(f)$ and $H_{\alpha}(v)$, defined by

$$
\begin{gathered}
E_{\alpha}(f)=\iint\left|v-v^{\prime}\right|^{\alpha} f\left(v^{\prime}\right) f(v) d v^{\prime} d v \\
H_{f, \alpha}(v)=\int_{v^{\prime}}\left|v-v^{\prime}\right|^{\alpha} f\left(v^{\prime}\right) d v^{\prime}
\end{gathered}
$$

We shall omit the dependency of $H$ with respect to $f$ when there are no ambiguity.

Properties 2.1.1 Then, we get

$$
\begin{gathered}
\partial_{f} E_{3}(f)=2 \int H_{3}(f, v) d v \\
\partial_{v} F=2 \int\left|v-v^{\prime}\right| f\left(v^{\prime}\right) d v^{\prime}=2 H_{1}(v), \\
3 \partial_{v} H_{3}=F .
\end{gathered}
$$


These properties can be easily checked, by splitting the integrals into $v^{\prime}>v$ and $v>v^{\prime}$.

The existence and uniqueness of an equilibrium state are given in [4]. The strict convexity of $\mathcal{E}$, follows from the following result that will be also useful to prove the entropy decay for an implicit scheme:

Lemme 2.2 For any function $f$ in

$$
\widetilde{\wp}=\left\{f:\left(1+|v|^{4}\right) f \in L_{1}(\mathbb{R}), \int f=0 \quad \text { and } \int v f(v) d v=0\right\},
$$

$E_{3}(f) \geq 0$.

Proof. Let $f \in \mathcal{C}_{0}^{\infty}$, and integrable. We have:

$$
H_{3}(f, v)=\int f\left(v^{\prime}\right)\left|v-v^{\prime}\right|^{3} d v^{\prime}=-\int f\left(v-v^{\prime}\right)\left|v^{\prime}\right|^{3} d v^{\prime}
$$

and, differentiating with respect to $v$

$$
\partial_{v} H_{3}(f, v)=3 \int f\left(v^{\prime}\right)\left|v-v^{\prime}\right|\left(v-v^{\prime}\right) d v^{\prime}=-\int \partial_{v} f\left(v-v^{\prime}\right)\left|v^{\prime}\right|^{3} d v^{\prime}
$$

which gives the following identity

$$
\partial_{v}^{2} H_{3}(f, v)=6 H_{3}(f, v)=-H_{3}\left(\partial_{v}^{2} f, v\right),
$$

Then,

$$
\begin{aligned}
E_{1}(f)=\int f(v) H_{1}(f, v) d v=\frac{1}{6} \int f(v) \partial_{v}^{2} H_{3}(f, v) d v \\
=-\frac{1}{6} \int \partial_{v} f(v) \partial_{v} H_{3}(h, v) d v^{\prime}=-\frac{1}{6} E_{3}\left(\partial_{v} f(v)\right) .
\end{aligned}
$$

Moreover $\partial_{v}^{2} H_{1}(f, v)=2 f(v), E_{1}\left(\partial_{v} f\right)=-\int f^{2} d v<0$, and then

$$
E_{1}\left(\partial_{v} f\right)=-\frac{1}{6} \int \partial_{v}^{2} f H_{3}\left(\partial_{v}^{2} f\right) d v=-\frac{1}{6} E_{3}\left(\partial_{v}^{2} f\right)
$$

so we have $E_{3}\left(\partial_{v}^{2} f\right)=\int f^{2}(v) d v>0$, and $\partial_{v}^{2} f \in \wp$.

Since for any $g \in \mathcal{C}^{\infty}$, with compact support and such that $\int g=0$ and $\int v g(v) d v=0$, we can find a function $f \in \mathcal{C}^{\infty}$, integrable and such that $g=\partial_{v}^{2} f$ thus, $E_{3}(g)=6 \int f^{2}(v) d v$. By density, the result hold for any function of $\widetilde{\wp}$.

For sake of simplicity, we omit the index and set $E=E_{3}$ and $H=H_{3}$ in the reminder. 


\subsection{Pure Granular}

We suppose $\sigma=0$ in (2.1), and let $f=f(t, v)$ be a function so that:

$$
\partial_{t} f=\partial_{v}(F f) .
$$

By the definition of $F$ we have

$$
\int_{v} F f d v=0
$$

We can write the weak formulation:

Proposition 2.3 Let $f$ be a solution of (2.11). Then, $f$ satisfies for all test functions $\phi$ :

$$
\begin{array}{r}
\int \partial_{t} f \phi=-\int \partial_{v} \phi F f=-\iint \partial_{v} \phi\left|v-v^{\prime}\right|\left(v-v^{\prime}\right) f(v) f\left(v^{\prime}\right) d v d v^{\prime} \\
=-\frac{1}{2} \iint\left(\partial_{v} \phi-\partial_{v^{\prime}} \phi^{\prime}\right)\left|v-v^{\prime}\right|\left(v-v^{\prime}\right) f(v) f\left(v^{\prime}\right) d v d v^{\prime}
\end{array}
$$

by symmetry of $v$ and $v^{\prime}$. We can obtain also the weak form

$$
\int \partial_{t} f \phi=-\frac{1}{2 \rho} \int\left(\partial_{v}(\phi)-\partial_{v^{\prime}}\left(\phi^{\prime}\right)\right)\left(F-F^{\prime}\right) f f^{\prime} d v d v^{\prime}
$$

These equations verify mass and momentum conservation, and entropy decay.

Proof. Taking respectively $\phi=1, v, v^{2}$ in (2.13), we get respectively mass and momentum conservation, and the temperature decay. For $\phi=v^{2}$ and $\rho=1$

$$
\begin{aligned}
\partial_{t} \int f \phi & =-\frac{1}{2} \iint\left(2 v-2 v^{\prime}\right)\left|v-v^{\prime}\right|\left(v-v^{\prime}\right) f(v) f\left(v^{\prime}\right) d v d v^{\prime} \\
& =-\iint\left|v-v^{\prime}\right|\left(v-v^{\prime}\right)^{2} f(v) f\left(v^{\prime}\right) d v d v^{\prime}=-E(f) .
\end{aligned}
$$

So $d_{t} \int v^{2} f \leq 0$ implies the temperature decay, and the equilibrium corresponds to $E=0$.

Moreover, using $3 \partial_{v} H=F$, and $d_{f} E=2 \int H$ we obtain for $\phi=3 H(v)$ :

$$
d_{t} E=3 \int \partial_{t} f H=-(1 / 2) \iint\left(F(v)-F\left(v^{\prime}\right)\right)\left|v-v^{\prime}\right|\left(v-v^{\prime}\right) f(v) f\left(v^{\prime}\right) d v d v^{\prime} .
$$

Note that $F$ is strictly increasing:

$$
\partial_{v} F=\int 2\left|v-v^{\prime}\right| f\left(v^{\prime}\right) d v^{\prime}>0 .
$$

Therefore $\left(F(v)-F\left(v^{\prime}\right)\right)\left(v-v^{\prime}\right) \geq 0$, and $d_{t} E \leq 0$. Thus we prove the decay of entropy). If we suppose that there is conservation, i.e. $d_{t} E=0$, as $F$ is strictly increasing, we get $f(v)=0$ and by the conservation of mass, $f(v)$ is of the form $\rho \cdot \delta(v-u)$. 


\subsection{Case of the granular immersed in a thermal bath}

Let us now consider the case $\sigma \neq 0$ in the equation (2.1). Let $M$ define as follows

$$
\partial_{v} M=\frac{-F}{\sigma} M
$$

i.e.

$$
M(v)=C \exp \left(\frac{-1}{3 \sigma} H(v)\right) .
$$

We rewrite the equation $(2.1)$

$$
\partial_{t} f=\partial_{v}\left(\sigma M \partial_{v}(f / M)\right)=\frac{\sigma}{\rho} \partial_{v}\left(\int_{v^{\prime}} f M \partial_{v}(f / M)-f M^{\prime} \partial_{v^{\prime}}(f / M)^{\prime} d v^{\prime}\right)
$$

where $\rho=\int f(v) d v$ denotes the mass and because the second term is zero by integrating by part and using 2.16 and 2.12 :

$$
\int M^{\prime} \partial_{v^{\prime}}(f / M)^{\prime} d v^{\prime}=-\int \partial_{v^{\prime}} M^{\prime}(f / M)^{\prime} d v^{\prime}=-\frac{1}{6 \sigma} \int F\left(v^{\prime}\right) f\left(v^{\prime}\right) d v^{\prime}=0
$$

Then, we can write the following weak symmetrized form of (2.1):

$$
\partial_{t} \int f \phi d v=\frac{-\sigma}{2 \rho} \iint\left(\partial_{v} \phi-\partial_{v^{\prime}} \phi^{\prime}\right) f f^{\prime}\left(\partial_{v} \log (f / M)-\partial_{v^{\prime}} \log (f / M)^{\prime}\right) d v d v^{\prime} .
$$

The mass and momentum conservation, as the decay of entropy, are obvious, by choosing $\phi=1, v, \log (f / M)$, respectively.

Note that the above formulation has a structure close to the Fokker-PlanckLandau one's, in the so called log form, that has been studied by the authors $[7,2]$. The only terms in the above expression that can be modified without changing the properties of the operator (conservation of mass, momentum and the decay of entropy) is the product $f f^{\prime}$.

\section{Discretization}

In this section, we introduce a discrete version of the equation (2.1), that degenerates correctly to the pure granular equation, when $\sigma \rightarrow 0$, i.e. "an asymptotic preserving scheme". Moreover, this scheme must conserve the properties of conservation of the mass, and momentum, as the decay of entropy.

We first deal with the pure granular case, which brings up the following problem: find a scheme that preserve the decay of entropy.

Initially designed for the Fokker-Planck linear equations, the method of ChangCooper (cf. [16]) allowed us to build such a discretization.

For all the different schemes, we consider an uniform grid in velocity:

$$
v_{i}=i \Delta v,
$$


for $i=1, N$. The macroscopic quantities are defined using standard quadrature formula: for the mass

$$
\rho=\sum_{i} f_{i} \Delta v
$$

the momentum

$$
\rho u_{f}=\sum_{i} f_{i} v_{i} \Delta v
$$

and the temperature

$$
T=\sum_{i} f_{i}\left(v-u_{f}\right)^{2} \Delta v
$$

\subsection{Case of pure granular: $\sigma=0$}

This section is devoted to the limiting case $\sigma=0$. We present two different methods.

\subsubsection{First method}

Let us first consider the more natural discretized version of the granular equation (2.11). The term $F$ is discretized using a standard quadrature formula

$$
F_{i}=\sum_{j}\left|v_{i}-v_{j}\right|\left(v_{i}-v_{j}\right) f\left(v_{j}\right) \Delta v
$$

This satisfies

$$
\sum_{i} F_{i} f_{i}=0
$$

by symmetry and $F_{i+1}-F_{i} \geq(\Delta v)^{2} \rho$ and thus it is strictly increasing. Indeed, as the grid is uniform, one gets:

$$
v_{i+1}-v_{i}=\Delta v, \quad\left|v_{i+1}-v_{j}\right|>\left|v_{i}-v_{j}\right|-\Delta v,
$$

thus

$$
\begin{gathered}
F_{i+1}-F_{i} \geq \Delta v\left(\sum_{j}\left|v_{i+1}-v_{j}\right| f_{j} \Delta v\right)-\Delta v \sum_{j}\left(v_{i}-v_{j}\right) f_{j} \Delta v \\
\geq \Delta v \sum_{j}\left(\left|v_{i+1}-v_{j}\right|-\left(v_{i}-v_{j}\right)\right) f_{j} \Delta v=\Delta v \sum_{j} K_{i, j} f_{j} \Delta v
\end{gathered}
$$

1. if $v_{i+1}>v_{j}$ then $K_{i, j}=\Delta v>0$,

2. if $v_{i+1}<v_{j}$ then $K_{i, j}=2 v_{j}-v_{i+1}-v_{i}=2\left(v_{j}-v_{i+1}\right)+\Delta v>\Delta v$. 
So we get the inequality $F_{i+1}-F_{i} \geq(\Delta v)^{2} \rho$.

We consider the following upwind scheme for this transport equation (in the velocity variable):

$$
\partial_{t} \sum_{i} f_{i} \phi_{i}=-\frac{1}{\Delta v} \sum_{i}\left(\left(\phi_{i+1}-\phi_{i}\right) F_{i}^{-}+\left(\phi_{i}-\phi_{i-1}\right) F_{i}^{+}\right) f_{i} .
$$

Let us mention that the $N$ terms $F_{i}$ can be computed in $\mathrm{O}(\mathrm{N})$ operations using a splitting of the sum into the $j$ before and after $v_{i}$ that reduce the complexity of their evaluation. Note also that there exists some index $i_{0}$ such that $F_{i}<0$ for all $i<i_{0}$ and $F_{i}>0$ for all $i>i_{0}$ (this index can move in time). Moreover, we have $F_{1}<0$ and $F_{N}>0$ and thus, no boundary condition are needed: we don't have to prescribe the value of $f_{0}$ of $f_{N+1}$.

The mass and momentum are conserved taking $\phi_{i}=1, v_{i}$ in (3.3) and using the condition (3.2). The evolution of the temperature is

$$
\frac{d T}{d t}=-6 E-(\Delta v) \sum_{i}\left(F_{i}^{-}-F_{i}^{+}\right) f_{i} .
$$

The second term of the right hand side is positive and one cannot conclude about the decay of the temperature. For the discrete entropy, E, the same conclusion holds.

However, discrete steady state for this scheme exists: consider state such that $i_{0}$ with $f_{i}=0$ for all $i>i_{0}+1$ or $i<i_{0}$. The values of the distribution function on the two non vanishing points and the value of $i_{0}$ are determined from mass and momentum conservation:

$$
f_{i_{0}+1}+f_{i_{0}}=\rho, \quad v_{i_{0}+1} f_{i_{0}+1}+v_{i_{0}} f_{i_{0}}=\rho u_{f} .
$$

\subsubsection{Second method}

We define the discrete analog of $H(v)$ and the entropy $E(2.5)$ by

$$
H_{i}=\frac{1}{3} \sum_{j}\left|v_{i}-v_{j}\right|^{3} f_{j} \Delta v
$$

and

$$
E=\frac{1}{2} \sum_{i} H_{i} f_{i} \Delta v
$$

A second discretization can be obtained in the pure granular equation. By integration by parts, we get:

$$
\int \phi \partial_{v}(F f) d v=-\iint \partial_{v} \phi(v)\left|v-v^{\prime}\right|\left(v-v^{\prime}\right) f(v) f\left(v^{\prime}\right) d v^{\prime} d v .
$$


The analogous discrete weak formulation is

$$
\partial_{t} \sum_{i} f_{i} \phi_{i} \Delta v=-(\Delta v)^{2} \sum_{i, j} D \phi_{i}^{j} f_{i} f_{j}\left|v_{i}-v_{j}\right|\left(v_{i}-v_{j}\right),
$$

where the finite difference operator at point $i$ is uncentered in a direction depending on the point $j$ as

$$
D \phi_{i}^{j}=\frac{\phi_{i+1}-\phi_{i}}{\Delta v} 1_{i<j}+\frac{\phi_{i}-\phi_{i-1}}{\Delta v} 1_{i>j} .
$$

This particular choice can be physically interpreted going back to a Boltzmann quasi-elastic monodimensionnal model.

First, a symmetry can be output of (3.5), to obtain

$$
\partial_{t} \sum_{i} f_{i} \phi_{i} \Delta v=-\frac{1}{2}(\Delta v)^{2} \sum_{i, j}\left(D \phi_{i}^{j}-D \phi_{j}^{i}\right) f_{i} f_{j}\left|v_{i}-v_{j}\right|\left(v_{i}-v_{j}\right) .
$$

Let us consider two particles with velocity $v_{i}$ and $v_{j}$ (with $i<j$ for example). The only elastic collision between these 2 particles consist in swapping the both, but this does not change the distribution function. If one allows slightly non elastic collisions but preserving the momentum, i.e. the postcollisional velocities have the same average (the particle have the same mass) as before the collision. Therefore, the less inelastic post-collisional velocities are $\left(v_{i+1}\right.$, $\left.v_{j-1}\right)$ and $\left(v_{i-1}, v_{j+1}\right)$.

To decrease the energy of the system is equivalent to decrease the relative velocity, so the velocities must get closer. The latter corresponds to a increasing of energy and is not physically relevant. The choice which corresponds to a minimal decrease of energy, is thus $\left(v_{i}, v_{j}\right) \mapsto\left(v_{i+1}, v_{j-1}\right)$ (for $i<j$ ).

Proposition 3.1 The scheme (3.6)-(3.5) preserves mass, and momentum, and the decay of energy and of entropy $E=\frac{1}{2} \sum_{i} H_{i} f_{i} d v$ with $H_{i}=\frac{1}{3} \sum_{j} \mid v_{i}-$ $\left.v_{j}\right|^{3} f_{j} \Delta v$.

Proof. Standing $(\phi=1)$ in (3.6), one gets mass conservation.

For momentum, let $\left(\phi_{i}=v_{i}\right)$, (3.6) write as $1_{i<j}+1_{i>j}$, and (3.5) is null. Using (3.7) with $\left(\phi_{i}=v_{i}^{2}\right)$ one gives:

$$
\begin{aligned}
2 \frac{d T}{d t}= & -(\Delta v)^{2} \sum_{i<j}\left(v_{i+1}^{2}+v_{j-1}^{2}-v_{i}^{2}-v_{j}^{2}\right) f_{i} f_{j}\left|v_{i}-v_{j}\right|\left(v_{i}-v_{j}\right) \\
& +(\Delta v)^{2} \sum_{i>j}\left(v_{i-1}^{2}+v_{j+1}^{2}-v_{i}^{2}-v_{j}^{2}\right) f_{i} f_{j}\left|v_{i}-v_{j}\right|\left(v_{i}-v_{j}\right) \leq 0 .
\end{aligned}
$$

For the entropy, one obtains:

$$
\begin{aligned}
2 \frac{d}{d t} E= & -(\Delta v)^{2} \sum_{i<j}\left(H_{i+1}-H_{i}-\left(H_{j}-H_{j-1}\right)\right) f_{i} f_{j}\left|v_{i}-v_{j}\right|\left(v_{i}-v_{j}\right) \\
& +(\Delta v)^{2} \sum_{i>j}\left(H_{i-1}+H_{j+1}-H_{i}-H_{j}\right) f_{i} f_{j}\left|v_{i}-v_{j}\right|\left(v_{i}-v_{j}\right) .
\end{aligned}
$$


Using the convexity of $w \rightarrow|w-a|^{3}$, one find that the sequence $H_{i+1}-H_{i}$ is increasing in $i$ or equivalently in $v_{i}$ and then

$$
\frac{d}{d t} E \leq 0
$$

Lemme 3.2 The system (3.5) has a global positive solution, for any positive initial data $\left(f_{i}^{0}>0\right.$, for all $\left.i\right)$.

Proof. The existence of a solution for this semi-discretized system can be easily obtained using a Cauchy-Lipschitz theorem for small time. This solution is global in time using a minoration of the solution by retaining only the loss term and the mass conservation (that provides an upperbound). Indeed, if $f$ is solution, the corresponding discrete velocities system for each $i$ is

$$
\begin{aligned}
\partial_{t} f_{i}= & (\Delta v)\left[f_{i-1} \sum_{i<j} f_{j}\left(v_{i-1}-v_{j}\right)^{2}+f_{i+1} \sum_{i>j} f_{j}\left(v_{i+1}-v_{j}\right)^{2}\right] \\
& -(\Delta v) f_{i} \sum_{j} f_{j}\left(v_{i}-v_{j}\right)^{2} .
\end{aligned}
$$

Moreover,

$$
(\Delta v) \sum_{j} f_{j}\left(v_{i}-v_{j}\right)^{2}<K
$$

where $K$ is a constant which depends of the length of the domain, and of the conserved quantities (mass and momentum). Thus, with $\sum_{i} f_{i}=\rho=$ constant, we get

$$
\rho \geq f_{i}(t) \geq f_{i}^{0} \exp (-K t) .
$$

Therefore, the maximal solution is global in time.

Equilibrium state for this scheme:

Lemme $3.3 \frac{d T}{d t}=0$ if and only if $f_{i}=0$ for $i<i_{0}$ and $i>i_{0}+1, i_{0}$ and $f_{i_{0}}, f_{i_{0}+1}$ are determined using mass and momentum conservation.

Proof. We start from (3.8). The converse is obviously true. For the direct implication, let $f$ be such that the right hand side of (3.8) is null, but with a fixed mass and momentum. Since the mass is not null, they necessary exist an index $i_{0}$ such that $f_{i_{0}}>0$. Since in the r.h.s. of (3.8), all the terms in factor of $f_{i} f_{j}$ are non positive except for $j=i-1$ or $j=i+1$, this leads to $f_{i}=0$ for $i<i_{0}-1$ and $i>i_{0}+1$. Now, since the term in factor of $f_{i_{0}-1} f_{i_{0}+1}$ is also negative thus one of $f_{i_{0}-1}$ and $f_{i_{0}+1}$ is null. Equation (3.4) determines $i_{0}$ and the value of $f_{i_{0}} f_{i_{0}+1}$.

If one compares the two schemes proposed here, the first one is uncentered at a "macroscopic level" that depends only on the integrated value of $F$ whereas, the second consists in a "microscopic" uncentered scheme where only the physically relevant collision are allowed. 


\subsection{Chang-Cooper method for a Fokker-Planck linear equation}

Before using the Chang-Cooper method for the granular media, we briefly recall this method on a more simple linear Fokker-Planck equation:

set $F=v$ in $(2.1)$,

$$
\partial_{t} f=\partial_{v}\left(v f+\sigma \partial_{v} f\right)
$$

that can be put under the form

$$
\partial_{t} f=\sigma \partial_{v}\left(M \partial_{v}(f / M)\right),
$$

where $M$ is a Maxwellian, and $M(v)=\exp \left(-|v|^{2} / 2 \sigma\right)$.

Originally, the method of Chang-Cooper was designed to preserve equilibrium state of Fokker-Planck equation. Another interesting feature of this method is its ability to degenerate correctly to an upwind scheme, for the equation of convection, when $\sigma \rightarrow 0$, with velocity grid fixed. Moreover, it's an entropy decaying method and not just an equilibrium state preserving method.

We set $M_{i}=M\left(v_{i}\right)$. This method can be written as

$$
\partial_{t} f_{i}=\frac{F_{i+1 / 2}-F_{i-1 / 2}}{\Delta v}
$$

with $F_{i+1 / 2}=\frac{\sigma}{\Delta v} \tilde{M}_{i+1 / 2}\left(\frac{f_{i+1}}{M_{i+1}}-\frac{f_{i}}{M_{i}}\right)$, where

$$
\tilde{M}_{i+1 / 2}=\frac{M_{i} M_{i+1}}{M_{i+1}-M_{i}}\left(\ln M_{i+1}-\ln M_{i}\right),
$$

thus

$$
F_{i+1 / 2}=\frac{\sigma}{\Delta v}\left(f_{i+1}-f_{i}\right)+\frac{\sigma}{\Delta v}\left(-1+\frac{\tilde{M}_{i+1 / 2}}{M_{i+1}}\right) f_{i+1}+\frac{\sigma}{\Delta v}\left(1-\frac{\tilde{M}_{i+1 / 2}}{M_{i}}\right) f_{i} .
$$

Analyze each term:

$$
\begin{aligned}
\frac{\sigma}{\Delta v}\left(1-\frac{\tilde{M}_{i+1 / 2}}{M_{i}}\right) & =\frac{\sigma}{\Delta v}\left(1-\frac{M_{i+1}}{M_{i+1}-M_{i}}\left(\ln M_{i+1}-\ln M_{i}\right)\right) \\
& =\frac{\sigma}{\Delta v} \ln \frac{M_{i+1}}{M_{i}}\left(\frac{1}{\ln M_{i+1}-\ln M_{i}}-\frac{M_{i+1}}{M_{i+1}-M_{i}}\right) \\
& =-\frac{\sigma}{\Delta v} \ln \frac{M_{i+1}}{M_{i}}\left(\frac{1}{\ln \frac{M_{i}}{M_{i+1}}}-\frac{1}{\left.\frac{M_{i}}{M_{i+1}}\right)}\right) .
\end{aligned}
$$

Setting $w=\ln \left(M_{i} / M_{i+1}\right)$, then we get:

$$
\begin{aligned}
\frac{\sigma}{\Delta v}\left(1-\frac{\tilde{M}_{i+1 / 2}}{M_{i}}\right)= & \frac{\sigma}{\Delta v} w\left(\frac{1}{w}-\frac{1}{\exp w-1}\right) \\
& =\frac{\sigma}{\Delta v} w h(w)=\frac{\sigma}{\Delta v} w \theta
\end{aligned}
$$


where $\theta=h(w)$ and $h$ is the function

$$
h(x)=\frac{1}{x}-\frac{1}{e^{x}-1} .
$$

Now, $h$ is positive on $\mathbb{R}$, decreasing and varies between 0 and 1 .

Similarly, the second term reads:

$$
\frac{\sigma}{\Delta v}\left(-1+\frac{\tilde{M}_{i+1 / 2}}{M_{i+1}}\right)=\frac{\sigma}{\Delta v} w h(-w),
$$

and $h(-w)=-h(w)+1$, thus

$$
\frac{\sigma}{\Delta v}\left(-1+\frac{\tilde{M}_{i+1 / 2}}{M_{i+1}}\right)=-\frac{\sigma}{\Delta v} w(\theta-1) .
$$

Then

$$
\begin{aligned}
F_{i+1 / 2}= & \frac{\sigma}{\Delta v}\left(f_{i+1}-f_{i}\right)+\frac{\sigma}{\Delta v}(-w(\theta-1)) f_{i+1}+\frac{\sigma}{\Delta v}(w \theta) f_{i} \\
& =\frac{\sigma}{\Delta v}\left(f_{i+1}-f_{i}\right)+\frac{\sigma w}{\Delta v}\left(\theta f_{i}+(1-\theta) f_{i+1}\right) .
\end{aligned}
$$

We get an "upwind" scheme, mixed with a " $\theta$-scheme": this is the ChangCooper method.

It is easy to verify that this discretization correspond to the discretization of the weak formulation of the FPL equation

$$
\partial_{t} f_{i} \phi_{i}=\sum_{i} \sigma(D \phi)_{i+1 / 2}\left(M_{i+1 / 2} D(f / M)_{i+1 / 2}\right)
$$

where $D g$ stands for the centered finite difference i.e. $D(g)_{i+1 / 2}=\left(g_{i+1}-\right.$ $\left.g_{i}\right) / \Delta v$ and the coefficients $M_{i+1 / 2}$ is an average of the value between $M_{i}$ and $M_{i+1}$ to be defined. Such type of scheme is by construction entropy decaying and provide the good equilibrium state, see [2]. By taking $M_{i+1 / 2}$ as

$$
M_{i+1 / 2}=\frac{M_{i} M_{i+1}}{M_{i+1}-M_{i}} \log \left(M_{i+1} / M_{i}\right)
$$

this gives the Chang-Cooper scheme.

This scheme degenerates toward an upwind scheme for $\partial_{t} f=\partial_{v}(v f)$ when $\sigma \rightarrow 0$. Indeed, the scheme (3.10) with the choice (3.11) reads:

$$
\sum_{i} \partial_{t} f_{i} \phi_{i}=\sum_{i} 2 \sigma\left(D^{*} \phi\right)_{i+1 / 2} \frac{\log \left(M_{i+1} / M_{i}\right)}{M_{i+1}-M_{i}}\left(f_{i+1} M_{i}-M_{i+1} f_{i}\right),
$$

using

$$
\sigma \log \left(M_{i+1} / M_{i}\right)=\frac{-v_{i+1}^{2}+v_{i}^{2}}{2}=-\Delta v v_{i+1 / 2}
$$


with $v_{i+1 / 2}=\left(v_{i+1}+v_{i}\right) / 2$. Moreover, for $i$ such that $v_{i}>0$

$$
\frac{M_{i}}{M_{i+1}-M_{i}} \rightarrow-1
$$

as $\sigma \rightarrow 0$ whereas it tends to 0 if $v_{i}<0$. Thus the limit of the scheme as $\sigma \rightarrow 0$ is

$$
\begin{gathered}
\partial_{t} f_{i}=v_{i+1 / 2}^{+}\left(f_{i+1}-f_{i}\right)+v_{i-1 / 2}^{-}\left(f_{i}-f_{i-1}\right) \\
\partial_{t} f_{i}=2 v_{i+1 / 2}\left(f_{i+1}-f_{i}\right) 1_{v_{i}>0}+2 v_{i-1 / 2}\left(f_{i}-f_{i-1}\right) 1_{v_{i-1}<0}
\end{gathered}
$$

denotes the positive/negative part of $x$ i.e. $x^{ \pm}=(x \pm|x|) / 2$.

\subsection{Discretization with $\sigma \neq 0$}

Taking the granular equation (2.1), we look for a discretization similar to the FPl one's, based on the Chang-Cooper method. Moreover, this scheme must preserve the properties of conservation and of decay, and degenerate to the equation of pure granular, when $\sigma \rightarrow 0$.

\subsubsection{A discretization based on the symmetric form}

We define the discrete analog of $H(v)$ and the entropy $\mathcal{E}(2.5)$ by

$$
H_{i}=\frac{1}{3} \sum_{j}\left|v_{i}-v_{j}\right|^{3} f_{j} \Delta v
$$

and

$$
\mathcal{E}=\sigma \sum_{i} f_{i} \ln \left(f_{i}\right) d v+\frac{1}{2} \sum_{i} H_{i} f_{i} \Delta v .
$$

We define also a discrete version of (2.17) by

$$
M_{i}=\exp \left(-\frac{H_{i}}{\sigma}\right) \text {. }
$$

From the $H_{i}$ 's we define discrete of $F$ by

$$
F_{i+1 / 2}=\frac{H_{i+1}-H_{i}}{\Delta v}
$$

and using the convexity of the function $w \rightarrow|w-a|^{3}$ one can verify easily that $F_{i+1 / 2} \geq F_{i-1 / 2}$ thus the sequence $F_{i+1 / 2}$ is increasing in $i$.

One can remark that by splitting the sum in two parts $\sum_{j \geq i}$ and $\sum_{j \leq i}$ than the $H_{i}$ 's can be evaluated in $\mathrm{O}(\mathrm{n})$ operations as explained by the authors in $([11,12])$ for a similar equation, the isotropic Fokker-Planck-Landau equation. 
In order to preserve mass momentum and the decay of the entropy we discretize the weak-symmetrized form (2.18),

$$
\partial_{t} \int f \phi d v=\frac{-\sigma}{2 \rho} \iint\left(\partial_{v} \phi-\partial_{v^{\prime}} \phi^{\prime}\right) f f^{\prime}\left(\partial_{v} \log (f / M)-\partial_{v^{\prime}} \log (f / M)^{\prime}\right) d v d v^{\prime},
$$

as follows

$$
\begin{aligned}
\frac{-\sigma}{2 \rho} \sum_{i} \sum_{j}\left(D \phi_{i+1 / 2}\right. & \left.-D \phi_{j+1 / 2}\right) f_{i+1 / 2} f_{j+1 / 2} \\
& \cdot\left(D \log (f / M)_{i+1 / 2}-D \log (f / M)_{j+1 / 2}\right)
\end{aligned}
$$

where $D g_{i+1 / 2}=\left(g_{i+1}-g_{i}\right) / \Delta v$ and the averaged value of the product $f_{i+1 / 2} f_{j+1 / 2}$ has to be defined.

On this form conservation of mass and mean velocity and the decay of the discrete entropy $\mathcal{E}$ are easily verified choosing $\phi=1, v, H$ respectively.

We shall now define the product $f_{i+1 / 2} f_{j+1 / 2}$ in such a way that, we will have the much simplest scheme as possible, that degenerate correctly when $\sigma$ go to 0 , and for which the collision term can be evaluated at the lower cost as possible, that is in $O(n)$. Moreover, approximating $f_{i+1 / 2} f_{j+1 / 2}$ by any positive formulae allows to insure conservation of the mass, the mean velocity and the decaying of the entropy. The first idea is to use the so called entropic average, see $([8])$, that permits to get rid of the log term

$$
f_{i+1 / 2} f_{j+1 / 2}=\left(\frac{(f / M)_{j} D(f / M)_{i+1 / 2}-(f / M)_{i} D(f / M)_{j+1 / 2}}{D \log (f / M)_{i+1 / 2}-D \log (f / M)_{j+1 / 2}}\right) M_{i+1 / 2} M_{j+1 / 2}
$$

Using this choice, one get

$$
\begin{aligned}
& \frac{-\sigma}{2 \rho} \sum_{i} \sum_{j}\left(D \phi_{i+1 / 2}-D \phi_{j+1 / 2}\right) M_{i+1 / 2} M_{j+1 / 2} \\
& \cdot\left((f / M)_{j} D(f / M)_{i+1 / 2}-(f / M)_{i} D(f / M)_{j+1 / 2}\right),
\end{aligned}
$$

that can be related to the non-log form of the Fokker-Planck equation:

$$
\begin{aligned}
(f / M)_{j} D(f / M)_{i+1 / 2} & -(f / M)_{i} D(f / M)_{j+1 / 2} \\
& =(f / M)_{j}(f / M)_{i+1}-(f / M)_{i}(f / M)_{j+1} .
\end{aligned}
$$

We define the product $M_{i+1 / 2} M_{j+1 / 2}$ in such way that the scheme reduces to a "good" scheme when $\sigma \rightarrow 0$, and using the analysis done for the linear Fokker-Planck equation, we choose

$$
C_{i j}=M_{i+1 / 2} M_{j+1 / 2}=\frac{M_{i+1} M_{j} M_{i} M_{j+1}}{M_{i+1} M_{j}-M_{i} M_{j+1}} \log \left(M_{i+1} M_{j} / M_{i} M_{j+1}\right) .
$$


The ODE system has the following structure

$$
\partial_{t} f_{i}=\sigma \sum_{j} C_{i j}\left(\frac{f_{i+1} f_{j}}{M_{i+1} M_{j}}-\frac{f_{j+1} f_{i}}{M_{j+1} M_{i}}\right)+C_{i-1, j}\left(\frac{f_{i-1} f_{j}}{M_{i-1} M_{j}}-\frac{f_{j+1} f_{i}}{M_{j+1} M_{i}}\right)
$$

with $C_{i j}$ given by $(3.15)$.

On this form, the cost to evaluate the coefficients of the differential system is quadratic.

In order to recover a linear cost, let us simplify the expression (3.15) for $M_{i+1} M_{j}>M_{j+1} M_{i}$ i.e. with $z_{j}=H_{j+1}-H_{j}+H_{i}-H_{i+1}>0$. One approximates

$$
\frac{1}{M_{j+1} M_{i}-M_{i+1} M_{j}}=\frac{1}{M_{j+1} M_{i}\left(1-\exp \left(-z_{j} / \sigma\right)\right)} \approx \frac{1}{M_{j+1} M_{i}}\left(z_{j} / \sigma\right) .
$$

Then,

$$
C_{i j} \approx M_{j} M_{i+1}\left(1+\frac{\sigma}{z_{j}}\right) \frac{z_{j}}{\sigma}
$$

and doing the same type of approximation for $j \leq i$ one obtain the discrete weak formulation

$$
\begin{gathered}
\sum_{i=0}^{n} \partial_{t} f_{i} \phi_{i}=-\frac{1}{2 \rho \Delta v} \sum_{i, j=0}^{n-1}\left(D \phi_{i+1 / 2}-D \phi_{j+1 / 2}\right)\left(\frac{f_{i+1} f_{j}}{M_{i+1} M_{j}}-\frac{f_{j+1} f_{i}}{M_{j+1} M_{i}}\right) \\
\cdot\left(M_{j+1} M_{i}\left(\sigma+z_{j}\right) \cdot 1_{\{j>i\}}+M_{i+1} M_{j}\left(\sigma-z_{j}\right) \cdot 1_{\{j<i\}}\right)
\end{gathered}
$$

and as $F_{j+1 / 2}-F_{i+1 / 2}:=\frac{H_{j+1}-H_{j}}{\Delta v}-\frac{H_{i+1}-H_{i}}{\Delta v}=\frac{z_{j}}{\Delta v}$, we deduce

$$
\begin{aligned}
\sum_{i=0}^{n} \partial_{t} f_{i} \phi_{i}= & -\frac{\sigma}{\rho \Delta v} \sum_{i, j=0}^{n-1} D \phi_{i+1 / 2}\left(M_{j+1} M_{i} \cdot 1_{\{j>i\}}+M_{i+1} M_{j} \cdot 1_{\{j<i\}}\right) \\
& \cdot\left(\frac{f_{i+1} f_{j}}{M_{i+1} M_{j}}-\frac{f_{j+1} f_{i}}{M_{j+1} M_{i}}\right)-\frac{1}{\rho} \sum_{i, j=0}^{n-1} D \phi_{i+1 / 2}\left(F_{j+1 / 2}-F_{i+1 / 2}\right) \\
& \cdot\left(M_{j+1} M_{i} \cdot 1_{\{j>i\}}-M_{i+1} M_{j} \cdot 1_{\{j<i\}}\right)\left(\frac{f_{i+1} f_{j}}{M_{i+1} M_{j}}-\frac{f_{j+1} f_{i}}{M_{j+1} M_{i}}\right) .
\end{aligned}
$$

Using the choice (3.17) for the coefficients $C_{i j}$, one gets a tridiagonal matrix: for $i=1, \ldots, n-1$

$$
\partial_{t} f_{i}=a_{i+1} f_{i+1}-\left(a_{i}+b_{i}\right) f_{i}+b_{i-1} f_{i-1},
$$

and the boundary terms are,

$$
\begin{gathered}
\partial_{t} f_{0}=a_{1} f_{1}-b_{0} f_{0} \\
\partial_{t} f_{n}=-a_{n} f_{n}+b_{n-1} f_{n-1},
\end{gathered}
$$


where the coefficients $a_{i}$, and $b_{i}$ are all positives and defined by the following relations, with, for $i=1, \ldots, n$ :

$$
\begin{gathered}
a_{i}=\frac{1}{\rho \Delta v}\left[\frac{M_{i-1}}{M_{i}}\left(\frac{\sigma}{\Delta v}-F_{i-1 / 2}\right) \sum_{j=i-1}^{n-1} \frac{M_{j+1}}{M_{j}} f_{j}+\left(F_{i-1 / 2}+\frac{\sigma}{\Delta v}\right) \sum_{j=0}^{i-1} f_{j}\right. \\
\left.+\frac{M_{i-1}}{M_{i}} \sum_{j=i-1}^{n-1} \frac{M_{j+1}}{M_{j}} f_{j} F_{j+1 / 2}-\sum_{j=0}^{i-1} f_{j} F_{j+1 / 2}\right]
\end{gathered}
$$

and for $i=0, \ldots, n-1$ :

$$
\begin{gathered}
b_{i}=\frac{1}{\rho \Delta v}\left[\left(\frac{\sigma}{\Delta v}-F_{i+1 / 2}\right) \sum_{j=i}^{n-1} f_{j+1}+\frac{M_{i+1}}{M_{i}}\left(F_{i+1 / 2}+\frac{\sigma}{\Delta v}\right) \sum_{j=0}^{i} \frac{M_{j}}{M_{j+1}} f_{j+1}\right. \\
\left.+\sum_{j=i}^{n-1} f_{j+1} F_{j+1 / 2}-\frac{M_{i+1}}{M_{i}} \sum_{j=0}^{i} \frac{M_{j}}{M_{j+1}} f_{j+1} F_{j+1 / 2}\right] .
\end{gathered}
$$

Note that the coefficients $a_{i}$ and $b_{i}$ can be evaluated in $O(n)$ steps.

From the equation (3.20)-(3.22), we note that the system can be written as a difference of flows:

$$
\partial_{t} f_{i}=G_{i+1 / 2}-G_{i-1 / 2}
$$

where $G_{i+1 / 2}=a_{i+1} f_{i+1}-b_{i} f_{i}$, the conservation of the mass is obvious.

Properties 3.3.2 This scheme conserves mass and momentum $\left(\phi_{i}=v_{i}\right)$ for the system (3.19), and decays of the discrete entropy $\mathcal{E}$.

Proof. By construction the approximation of $f_{i+1 / 2} f_{j+1 / 2}$ is positive. Thus starting from (3.12) if we set $\phi_{i}=1$ and $v_{i}$ respectively we obtain the conservation of the mass $\rho$ and the mean velocity $u$.

For the entropy, as for the continuous model we have

$$
\frac{d}{d t} \mathcal{E}=\sum_{i} \frac{d f_{i}}{d t}\left(\ln f_{i}+\frac{H_{i}}{\sigma}\right)=\sum_{i} \frac{d f_{i}}{d t} \ln \left(\frac{f_{i}}{M_{i}}\right) .
$$

Thus by taken $\phi_{i}=\ln \left(\frac{f_{i}}{M_{i}}\right)$ in (3.12) we have evidently $\frac{d}{d t} \mathcal{E} \leq 0$.

If $f$ is such that $f_{i}=M_{i}$ thus we have $\frac{d}{d t} \mathcal{E}=0$. For the existence of such equilibrium, we refer to [4], their proof remains valid if we have a discrete measure instead of the Lebesgue measure. 


\subsubsection{Limit when $\sigma \rightarrow 0$ : a third discretization}

If one considers the limit $\sigma \rightarrow 0$ in the scheme (3.20-3.24), one gets the form based on the weak formulation $f f^{\prime}\left(F^{\prime}-F\right)$ instead of $\left|v-v^{\prime}\right|\left(v-v^{\prime}\right)$.

This scheme degenerates correctly when $\sigma \rightarrow 0$, indeed, $z_{j}$ is positive, defined by

$$
\exp \frac{-z_{j}}{\sigma}=\frac{M_{j+1} M_{i}}{M_{j} M_{i+1}} .
$$

So, when $\sigma \rightarrow 0, \exp \frac{-z_{j}}{\sigma} \rightarrow 0$ and the scheme becomes

$$
\begin{aligned}
& \sum_{i=0}^{n} \partial_{t} f_{i} \phi_{i} \Delta v=-\frac{1}{2 \rho} \cdot \sum_{i, j=0}^{n-1}\left(D \phi_{i+1 / 2}-D \phi_{j+1 / 2}\right) \\
& \cdot\left(F_{i+1 / 2}-F_{j+1 / 2}\right)\left(f_{j+1} f_{i} \cdot 1_{\{j>i\}}+f_{i+1} f_{j} \cdot 1_{\{j<i\}}\right)(\Delta v)^{2},
\end{aligned}
$$

which is a discrete form of the weak symmetrized form (2.14) of the pure granular operator.

Properties 3.3.4 The system limit conserves mass and momentum, and we have the decay of energy and of the discrete entropy. Equilibrium states are such that $f_{i}=0$ for $i<i_{0}$ and $i>i_{0}+1$, and $i_{0}, f_{i_{0}}, f_{i_{0}+1}$ are determined from mass and momentum, see 3.4.

Proof. By taking $\phi=1, v$ in (3.25), mass and momentum conservation are obtained. The sequence $F_{i+1 / 2}$ is increasing in $i$ and taking $\phi=v^{2}$ we have the decay of the temperature

$$
\begin{aligned}
\partial_{t} T=- & \frac{\Delta v}{\rho} \cdot \sum_{i, j=0}^{n-1}\left(v_{i+1 / 2}-v_{j+1 / 2}\right) \\
& \cdot\left(F_{i+1 / 2}-F_{j+1 / 2}\right)\left(f_{j+1} f_{i} \cdot 1_{\{j>i\}}+f_{i+1} f_{j} \cdot 1_{\{j<i\}}\right)(\Delta v)^{2},
\end{aligned}
$$

where $v_{i+1 / 2}=\frac{v_{i}+v_{i+1}}{2}$. The sequence $v_{i+1 / 2}$ is also increasing in $i$ and then all the terms $\left(v_{i+1 / 2}-v_{j+1 / 2}\right)\left(F_{i+1 / 2}-F_{j+1 / 2}\right)$ are non negative, which gives the result.

For the entropy we have

$$
\partial_{t} E=-\frac{1}{\rho} \cdot \sum_{i, j=0}^{n-1}\left(F_{i+1 / 2}-F_{j+1 / 2}\right)^{2}\left(f_{j+1} f_{i} \cdot 1_{\{j>i\}}+f_{i+1} f_{j} \cdot 1_{\{j<i\}}\right)(\Delta v)^{2} .
$$

Concerning the equilibrium, we start from the entropy production term (3.27). First let us verify that the sequence $F_{i+1 / 2}$ is a strictly increasing sequence in $i$. By the definition of $F_{i+1 / 2}$ we have

$$
\begin{aligned}
F_{i+1 / 2}-F_{i-1 / 2} & =\frac{1}{\Delta v}\left(H_{i+1}+H_{i-1}-2 H_{i}\right) \\
& =\frac{1}{\Delta v} \sum_{j} f_{j}\left(\left|v_{i+1}-v_{j}\right|^{3}+\left|v_{i-1}-v_{j}\right|^{3}-2\left|v_{i}-v_{j}\right|^{3}\right) .
\end{aligned}
$$


On can verify easily that we have the lowerbound

$$
\left(\left|v_{i+1}-v_{j}\right|^{3}+\left|v_{i-1}-v_{j}\right|^{3}-2\left|v_{i}-v_{j}\right|^{3}\right) \geq(\Delta v)^{2}
$$

so that

$$
F_{i+1 / 2}-F_{i-1 / 2} \geq(\Delta v)^{2} \rho .
$$

Consider $f$ such that the right hand side of (3.27) is null. Since the mass $\rho$ of $f$ is not null there exist $i_{0}$ such that $f_{i_{0}}>0$. All the terms involving in the right hand side of (3.27) are null since they have all the same sign. Using then (3.28) we obtain that $f_{j}=0$ for $j>i_{0}+1$ and $j<i_{0}-1$. And $i=i_{0}-1$ and $j=i_{0}$ gives $f_{i_{0}+1} f_{i_{0}-1}=0$. In other words, one of the both terms is null. That shows that $f$ is as we have claimed it: the values of $i_{0}, f_{i_{0}}$ and $f_{i_{0}+1}$ are uniquely determined by the values of the mass and the momentum.

\subsection{Time discretization}

We shall present the time discretization of system in the form (3.20)-(3.22).

\subsubsection{Explicit scheme}

In the explicit case, the system can be written:

$$
\begin{gathered}
f_{i}^{n+1}-f_{i}^{n}=\Delta t\left(a_{i+1}^{n} f_{i+1}^{n}-c_{i}^{n} f_{i}^{n}+b_{i-1}^{n} f_{i-1}^{n}\right) \\
f_{i}^{n+1}-f_{i}^{n}=\Delta t Q^{n} f_{i}^{n}
\end{gathered}
$$

i.e.

$$
f^{n+1}=\left[I d+\Delta t\left(\begin{array}{cccccc}
-c_{0}^{n} & a_{1}^{n} & 0 & \ldots & \ldots & 0 \\
b_{0}^{n} & -c_{1}^{n} & a_{2}^{n} & 0 & \ldots & 0 \\
0 & \ddots & \ddots & \ddots & & \vdots \\
\vdots & & \ddots & \ddots & \ddots & 0 \\
0 & \ldots & 0 & b_{n-2}^{n} & -c_{n-1}^{n} & a_{n}^{n} \\
0 & \ldots & \ldots & 0 & b_{n-1}^{n} & -c_{n}^{n}
\end{array}\right)\right] f^{n}
$$

where $c_{i}^{n}=a_{i}^{n}+b_{i}^{n}$.

A sufficient condition to have a positive matrix is to take $\Delta t$ satisfying $1-c_{i} \Delta t>0$ for all $i$. Therefore, we choose $\Delta t_{\max }=\left(\text { sup } c_{i}\right)^{-1}$.

Notice that $\Delta t_{\max }$ behave like $O\left(\frac{\Delta v^{2}}{\sigma+(\Delta v) \sup _{i}\left|F_{i+1 / 2}\right|}\right)$.

The properties of conservation of mass and momentum, of the system can be checked easily $\left(\phi_{i}=1, v_{i}\right)$. 


\subsubsection{Implicit scheme}

The implicit scheme reads:

$$
f_{i}^{n+1}-f_{i}^{n}=\Delta t a_{i+1}^{n+1} f_{i+1}^{n+1}-\Delta t c_{i}^{n+1} f_{i}^{n+1}+\Delta t b_{i-1}^{n+1} f_{i-1}^{n+1},
$$

i.e.

$$
f^{n}=\left(I d-\Delta t Q\left(f^{n+1}\right)\right) f^{n+1},
$$

where $Q\left(f^{n+1}\right)$ is the previous matrix, at range $n+1$, instead of $n$. We use an iterative method:

$$
f^{n}=\left(I d-\Delta t Q\left(f^{n}\right)\right) f^{n+1}
$$

and we define the process as:

$$
\begin{gathered}
\left(I d-\Delta t Q\left(g^{p}\right)\right) g^{p+1}=f^{n} \\
g^{0}=f^{n}
\end{gathered}
$$

to resolve it, where $g^{p}$ is the $p^{\text {ieme }}$ iterate.

At each iteration, mass and positivity are preserved. The conservation of mass follows from structure of the matrix $Q$, which corresponds to a conservative scheme. The last point follow from a classical algebra result [6]:

Let $N$ be a matrix $\in \mathbb{R}^{n} \times \mathbb{R}^{n}$, tridiagonal, whose diagonal coefficients are positives, the others negatives. Then $N^{-1}$ is positive, if and only if, there exists a diagonal positive matrix $D$, such that $D^{-1} N D$ is diagonal dominant. $N=\left(I d-\Delta t Q\left(g^{p}\right)\right)$, take $G \in \operatorname{Ker}(Q)$ positive which is always possible, then $N \widetilde{G}=\widetilde{G}$, where $\widetilde{G}$ is the diagonal positive matrix formed with the coefficients of $G$. Thus $\widetilde{G}^{-1} N \widetilde{G}=I d$ which is a diagonal dominant matrix. This concludes the proof.

One can also notice that if $f^{n}$ is an equilibrium then $g^{p}=f^{n}$.

Lemme 3.4 For the implicit scheme the entropy is decaying.

Proof. The entropy $\mathcal{E}$ can be written as:

$$
\mathcal{E}(f)=\sigma \int\left(f \ln f+\frac{1}{6} \int\left|v-v^{\prime}\right|^{3} f(v) f\left(v^{\prime}\right) d v^{\prime}\right) d v=\sigma \int(\ln f-\ln N) f d v,
$$

where $N(v)=\exp \left(-\frac{1}{6 \sigma} \int\left|v-v^{\prime}\right| f\left(v^{\prime}\right) d v^{\prime}\right)=\exp (-3 H(v) / 2 \sigma)$. We get

$$
\begin{aligned}
\mathcal{E}^{n+1}-\mathcal{E}^{n} & =\sigma\left(\int f^{n+1} \ln \left(\frac{f}{N}\right)^{n+1} d v-\int f^{n} \ln \left(\frac{f}{N}\right)^{n} d v\right) \\
& =\sigma\left(\int f^{n}\left(\ln \left(\frac{f}{N}\right)^{n+1}-\ln \left(\frac{f}{N}\right)^{n}\right) d v+\Delta t \int Q^{n+1} f^{n+1} \ln \left(\frac{f}{N}\right)^{n+1} d v\right),
\end{aligned}
$$

with $Q^{n+1}=Q\left(f^{n+1}\right)$,

$\mathcal{E}^{n+1}-\mathcal{E}^{n} \leq \sigma\left(\int f^{n} \ln \left(\frac{f^{n+1}}{f^{n}}\right)-\int f^{n} \ln \left(\frac{N^{n+1}}{N^{n}}\right) d v+\Delta t \int Q^{n+1} f^{n+1} \ln (N)^{n+1} d v\right)$. 
Using the entropy decay on the weak form (2.18) with $\phi=\ln (f / M)\left(M=N^{2}\right)$, one gets

$$
\begin{aligned}
\mathcal{E}^{n+1}-\mathcal{E}^{n} & \leq \sigma\left(\int_{v} f^{n} \ln (N)^{n} d v+\int_{v} f^{n+1} \ln (N)^{n+1} d v-2 \int_{v} f^{n} \ln (N)^{n+1} d v\right) \\
& \leq \sigma\left(\int_{v} f^{n}\left(\ln (N)^{n}-\ln (N)^{n+1}\right) d v+\int_{v}\left(f^{n+1}-f^{n}\right) \ln (N)^{n+1} d v\right) .
\end{aligned}
$$

By definition of $N$, we've:

$$
\begin{aligned}
\mathcal{E}^{n+1}-\mathcal{E}^{n} & \leq \frac{1}{2} \int_{v} \int_{v^{\prime}}\left|v-v^{\prime}\right|^{3}\left[f^{n}\left(f^{\prime n+1}-f^{\prime n}\right)-f^{\prime n+1}\left(f^{n+1}-f^{n}\right)\right] d v d v^{\prime} \\
& \leq-\frac{1}{2} \int_{v} \int_{v^{\prime}}\left|v-v^{\prime}\right|^{3}\left[\left(f^{\prime n+1}-f^{\prime n}\right)\left(f^{n+1}-f^{n}\right)\right] d v d v^{\prime}
\end{aligned}
$$

Since from lemma (2.2) the application

$$
g \rightarrow \iint g(v) g\left(v^{\prime}\right)\left|v-v^{\prime}\right|^{3} d v d v^{\prime}
$$

is positive for functions $g$ such that $\int g=\int v g=0$, we have the decay of entropy.

The proof for the discrete formulation follow the same lines:

$\mathcal{E}^{n+1}-\mathcal{E}^{n} \leq \sigma\left(\sum_{i} f_{i}^{n}\left(\ln \left(N_{i}\right)^{n}-\ln \left(N_{i}\right)^{n+1}\right) \Delta v+\sum_{i}\left(f_{i}^{n+1}-f_{i}^{n}\right) \ln \left(N_{i}\right)^{n+1} \Delta v\right)$.

The definition of $N$ gives:

$$
\mathcal{E}^{n+1}-\mathcal{E}^{n} \leq-\frac{1}{2} \sum_{i} \sum_{j}\left|v_{i}-v_{j}\right|^{3}\left[\left(f_{j}^{n+1}-f_{j}^{n}\right)\left(f_{i}^{n+1}-f_{i}^{n}\right)\right](\Delta v)^{2}
$$

By a discrete version of the lemma (2.2), which can be obtained by regularizing the sum of Dirac mass, we conclude to the decay of entropy.

\section{Numerical results}

We illustrate the method on two tests, a Dirac and a Gaussian for initial data. For the two tests the velocity domains is $[0,12.5]$, the total mass is one and the two initial data are centered at $v=4$. For the gaussian the variance is 1 . For all, the run of the time step is taken equals to 0.1. We use two uniform grids of 50 and 200 points. For the explicit scheme, we use sub cycling technic to respect the CFL constraint that guarantee positivity of the scheme. For the implicit scheme the iterative process is stopped when the error on the relative velocity is smaller than $10^{-12}$. 


\subsection{Computational cost}

Let us first compare the computational cost between explicit and implicit. The costs correspond to $\sigma=1$.

For the implicit scheme the number of iterates varies from 12, at the first time step, to 1 after few time steps when starting from the gaussian, and from 5 to 1 when starting from the Dirac, and this with the two grids. The number of iterates seems to does not depend of the number of points. For the explicit scheme, the number of sub cycles is nearly constant during the computation. Starting from the gaussian or the Dirac we need 60 subcycles with 50 points and 283 subcycles with 200 points. The cost of the iterations is the same for the implicit and the explicit.

More the grid is fine, larger is the cost ratio explicit/implicit. For very coarse grid (ten points) the cost of the explicit scheme when starting from the gaussian is smaller than the cost of the implicit but only for the first time steps of the relaxation.

One interesting feature of the implicit scheme concerns it's ability to reach an equilibrium state, despite the fact that the iterative process is stopped, when the error on the relative velocity is smaller than $O\left(10^{-n}\right)$. This implementation of the implicit scheme, strictly speaking, is not conservative for the mean velocity, but we observe that for any value of the allowed error, in long time, the scheme converge toward an equilibrium state for which, the mean velocity is nearly those of the initial condition up to an error of $O\left(10^{-n}\right)$. We plot the difference between the average velocity after total relaxation and the initial one, in function of the error, for the two types of initial condition, a Dirac mass and a Gaussian, see Figure (1).

Figure 1: Gaussian and Dirac initial condition: difference between the average velocity after total relaxation and the initial one in function of the error, in log scale 


\subsection{Graphics}

Figure (2) shows the evolution in time of the temperature starting from the Dirac mass, with 50 meshes, $\sigma=1$ and explicit and implicit scheme.

Figure (3) shows the evolution of the temperature starting from the Gaussian, with 50 and 200 grid points and again with $\sigma=1$ and using explicit and implicit version of the scheme.

Starting from the Gaussian, we can compare for $\sigma=1$ and $\sigma=0.00001$ the evolution of the distribution function on the figure (4) and the relaxation of the temperature (6). Implicit scheme is used with 50 grid points.

We can also show on figure (5), the relaxation of the temperature for the two initial condition when $\sigma=1$, using 50 grid points and implicit scheme.

Figure 2: Initial condition: Dirac mass. Evolution of the temperature and the entropy, 50 points, $\sigma=1$.

Figure 3: Initial condition: Gaussian. Evolution of the temperature and the entropy, 50 and 200 points, $\sigma=1$.

To finish let us show the behavior of the equilibrium state $f_{\infty}$ : we compare the gaussian $\exp \left(-v^{2} / 2\right)$ with the equilibrium state obtained with 
Figure 4: Initial condition: Gaussian. Evolution of the distribution function, 50 points, $\sigma=1$ and $\sigma=10^{-5}$.

Figure 5: Evolution of the temperature starting from Dirac mass and Gaussian, $\sigma=1,50$ points.

this initial data and corresponding to $\sigma=1$ and which behave for large $|v|$ as $\exp \left(-a|v|^{3}\right)$. We plot $-\log f$ in function of $v$, in logarithmic scale, see Figure 7. It is is easily seen on Figure ( 7 that for large velocity, the distribution function has the expected behavior, the slope of $-\log f(v)$ tends to 3 for the steady states of granular media, and to to for maxwellian.

\section{Conclusion}

In 1D, without friction term, the Chang-Cooper method allows us to construct a discretization for the granular equation.

Moreover, the discretization is performed at a linear cost in the number of grid points. An implicit scheme has been used. It converges in large time to the equilibrium state described in [4], and it preserves the properties of the equa- 
Figure 6: Evolution of the temperature starting from Gaussian with different values of the temperature of the thermal bath, $\sigma=1$ and $\sigma=10^{-5}$, 50 points.

Figure 7: The distribution function in log scale for a gaussian and the equilibrium state associated for $\sigma=1$.

tion. Moreover, the cost of such implicit scheme is less than the explicit one. This implicit method could also be used for the numerical methods described in $([11,12])$ for the isotropic Fokker-Planck-Landau equation.

In this study, the friction term was ignored. Since the discretization's process is applied to a symmetric form of the equation, the friction term cannot be included within the presented framework.

However, it should be possible to take it into account using splitting technics. 


\section{References}

[1] A. Baldassarri, A. Puglisi, and U. Marconi, Kinetics models of inelastic gases, Math. Models Meth. Appl. Sci., 12, p.965-984, 2002.

[2] D. Benedetto, E. Caglioti, The collapse phenomenon in one -dimensional inelastic point particle systems, Physica D, Vol. 132, p 457, 1999

[3] D. Benedetto, E. Caglioti, M. Pulvirenti, A kinetic equation for granular media, Math. Mod. and Num. Anal, M2AN, Vol 31, N 5, p 615, 1997.

[4] D. Benedetto, E. Caglioti, J.A. Carillo, M. Pulvirenti, A non-Maxwellian equilibrium distribution for the one-dimensional granular media, J. Stat. Phys., 91(5/6) p. 979, 1998.

[5] D. Benedetto, E. Caglioti, F. Golse, M. Pulvirenti, A hydrodynamical model arising in the context of granular media, Computers $\mathscr{E}$ Mathematics with Applications, V. 38, Issues 7-8, p. 121-131, October 1999.

[6] A. Berman, R.J. Plemmons, Nonnegative matrices in the mathematical sciences, Classics in Applied Mathematics, 1994

[7] C. Buet, S. Cordier, P. Degond, M. Lemou, Fast algorithms for numerical, conservative, and entropy approximations of the Fokker-Planck-Landau equation, J. Comput. Phys. 133, No.2, p. 310-322, 1997.

[8] C. Buet, S. Dellacherie, R. Sentis, Numerical solution of an ionic FokkerPlanck equation with electronic temperature, SIAM J. Numer. Anal., 39, No.4, 1219-1253, 2001.

[9] C. Buet and S. Cordier, Numerical analysis of conservative and entropy schemes for the Fokker-Planck-Landau equation, SIAM J. Numer. Anal., vol 36, No 3, p. 953-973, 1998.

[10] C. Buet and S. Cordier, Numerical method for the Compton scattering operator, to appear in Lecture Notes on the Discretization of the Boltzmann Equation World Scientific, ed. by N. Bellomo and R. Gatignol, 2003.

[11] C. Buet and S. Cordier, Conservative and entropy decaying numerical scheme for the isotropic Fokker-Planck-Landau equation, J. Comput. Phys. 145, No.1, 228-245 1998.

[12] C. Buet and S. Cordier, Numerical analysis of the isotropic Fokker-PlanckLandau equation. (English) J. Comput. Phys. 179, No.1, 43-67 2002.

[13] J.A. Carillo, On a 1-D granular media immersed in a fluid, preprint TMR, 2000 .

[14] J.A. Carillo, C. Cercignani, I.M. Gamba, Steady States of a Boltzmann equation for driven Granular media, Preprint.

[15] C. Cercignani, R. Illner, M. Pulvirenti, The mathematical theory of dilute gases, Appl. Math. Sc., 106, Springer Verlag, 1994. 
[16] J.S. Chang, G. Cooper, A practical difference scheme for Fokker-Planck equations, J. Comput. Phys. 6, p 1-16, 1970.

[17] M. Esteban and B. Perthame, On the modified Enskog equation for elastic and inelastic collisions. Models xith spin, Ann. Inst. Poincaré, 8 p.289308, 1991.

[18] I. Goldhirsch, G. Zanetti, Clustering instability in dissipative gases, Phys. Rev. Lett. 70, p. 1619, 1993.

[19] I. Goldhirsch, Scales and kinetics of granular flows, Chaos 9 p. 659-672, 1999.

[20] D. Görsch, Generalized discrete velocity models, Math. Models Meth Appl. Sci., 12, p. 49-76, 2002.

[21] E.W. Larsen, C.D. Levermore, G.C. Pomraning, J.G. Sanderson, Discretization methods for one-dimensional Fokker-Planck operators, $J$. Comput. Phys. 61, p. 359-390, 1985.

[22] S. Mac Namara, W.R. Young, Inelastic collapse and clumping in a onedimensional granular medium, Phys of Fluids, A4, Vol 3, p. 496, 1992.

[23] S. Mac Namara, W.R. Young, Kinetics of a one-dimensional granular medium in the quasi-elastic limit, Phys of Fluids, A5, Vol 1, p. 1619, 1993.

[24] S. Mac Namara, W.R. Young, Inelastic collapse in two dimension, Phys. Rev. E, 50, R28, 1994.

[25] G. Toscani, One dimensional kinetic models of granular flows, M2 AN, Vol34, 6, 2000. 